\title{
Enzymes of Phanerochaete chrysosporium Burds. and Irpex lacteus (Fr.)Fr.: Decolorization of Dyes and Effluents
}

\author{
R. Shrivastava, V. Christian, E' B. R. M. Vyas \\ Department of Biosciences, Saurashtra University, Rajkot 360 005, India.
}

White-rot Basidiomycetes (WRB) play a central role in global carbon cycles as a result of their innate ability to mineralize the lignin, which has a complex polymeric structure. Shallow stationary cultures of Phanerochaete chrysosporium, grown on low-nitrogen mineral medium, produced manganese peroxidase $(\mathrm{MnP})$ and lignin peroxidase $(\mathrm{LP})$. Sulphonphthalein (SP) dyes were decolorized by $\mathrm{MnP}$ activity. Decolorization of SP dyes occurred optimally at $\mathrm{pH}$ 4.0. An increase in the halogenation (bromine group) of the SP dyes decreases its substrate specificity for $\mathrm{MnP}$. The presence of additional auxochromes (position, type, and number) on the SP dye chromophore influence the suitability of $\mathrm{SP}$ dyes as an $\mathrm{MnP}$ substrate.

The methyl group in the ortho position (in the case of $o$-cresol red) is favored over that in meta position (in the case of $m$-cresol purple). Oxygen scavenger (sodium metabisulfite) and hydroxyl radical scavengers (thiourea and mannitol) did not have any influence on $\mathrm{MnP}$ decolorization activity. EDTA, a metal chelator, inhibited MnP-catalyzed decolorization reaction. The results highlight the SP dyes as substrates of $\mathrm{MnP}$ and provide another class of chromogen for the detection and estimation of ligninolytic peroxidases. Also, the results emphasize as a model for the bioremediation program of the structurally similar xenobionts and recalcitrant compounds stressing over enzyme(s) of white rot basidiomycete P. chrysosporium as a potent biochemical tool.

In another study, white-rot basidiomycete $I r-$ pex lacteus decolorized the textile dyes on solid medium. Decolorization on solid medium was of older mycelium. Extracellular enzyme extract prepared from I. lacteus-infested wheat straw possessed ligninolytic enzymes manganese peroxidase $(\mathrm{MnP})$, manganese-independent peroxidase (MIP), and lignin peroxidase (LP). Decolorization of triphenyl methane dyes is attributed to $\mathrm{MnP}$ activities.

The same color dyes (red, orange, and blue) were decolorized variably on the solid medium as well as by the ligninolytic enzymes of I. lacteus. The presence of a halogenated compound, cyanuric chloride, in the structure of reactive orange 13 makes it a suitable substrate for MIP, whereas $\mathrm{MnP}$ decolorizes reactive orange 16 that has a naphthalene ring with an hydroxyl group, presenting the dye as a simpler phenolic moiety. Decolorization of triphenyl methane dyes methyl blue (MB) and fast green (FG) was attributed to $\mathrm{MnP}$ activities. Brilliant green $(\mathrm{BG})$ resisted decolorization by ligninolytic activities. The presence of two 3-[2-(ethyl amino) ethyl] benzene sulfonate groups in FG and three 4-amino benzene sulfonate groups in MB favor the decolorization, whereas the presence of two $N, N$ diethyl amine groups in BG resist the decolorization by ligninolytic activities. Enzymatic decolorization of effluents led to the complete loss of the peak in the UV-region. Ligninolytic activities associated to decolorization of effluents showed no influence of $\mathrm{pH}$ and decolorized the effluent over the $\mathrm{pH}$ range of 3-5. The results highlight the efficacies of the ligninolytic system in designing a modest bioremediation program and also provide the means to distinguish among the peroxidases generated as a part of the ligninolytic system. 